\title{
ELECTRE TRI aplicado a avaliação da satisfação de consumidores
}

\author{
Helder Gomes Costa \\ UFF \\ André Fernando Uébe Mansur \\ CEFET-Campos \\ André Luís Policani Freitas \\ UENF \\ Rogério Atem de Carvalho \\ CEFET-Campos
}

\begin{abstract}
Resumo
As abordagens usuais para conhecer a percepção do consumidor quanto ao desempenho de serviços baseiam-se em impressões do consumidor (julgamentos subjetivos) à luz de múltiplos critérios. Em geral estas abordagens consideram uma função de agregação do tipo média ponderada, que apresenta efeitos compensatórios indesejáveis. O presente trabalho explora a aplicação do Auxílio Multicritério à Decisão, mais especificamente no método ELECTRE TRI, à classificação ordenada da satisfação dos consumidores. Isto adapta uma modelagem de apoio à decisão para um problema de diagnóstico do grau de satisfação. Um tratamento inédito para a estimativa dos parâmetros $p$ e $q$ (limites de preferência e de indiferença, respectivamente) é incorporado a modelagem, incorporando maior robustez ao uso de um método de AMD monodecisor na resolução de um problema multidecisor. Trata-se de forma inédita a questão de incomparabilidade, destacando que nem sempre a mesma é originada por imperícia na modelagem ou nas avaliações de desempenho. Uma importante diferença em relação aos métodos tradicionais de avaliação de desempenho é a independência do emprego do resultado em relação ao valor numérico da escala de julgamento de desempenho. A aplicação desta abordagem proposta é experimentada na avaliação da satisfação dos consumidores de uma bombonnière instalada em um shopping center.
\end{abstract}

Palavras-chave

Satisfação, qualidade, ELECTRE, multicritério, avaliação, MCDA.

\section{ELECTRE TRI applied to costumers satisfaction evaluation}

\begin{abstract}
Usual approaches to identify costumer's perceptions about service are based on subjective evaluations under multiple criteria. Generally, an aggregation function based on weighted average method is used to solve this type of problem, in despite of the compensatory problems that it causes. Present work explores the application of an MCDM based method in order to solve this problem. An original approach to estimates $p$ and q parameters in ELECTRE TRI is inserted on the model building, incorporating robustness to the proposal. Also, an original approach to incomparability's analyses is observed. An application of the proposal is experimented in a candy shop to evaluate consumer's satisfaction.
\end{abstract}

Key words

Satisfaction, quality, ELECTRE, multicriteria, evaluation, MDCA. 


\section{INTRODUĈ̣̃O}

Conforme reportado em Boljwin e Kumpe (1990), a competitividade está fundamentada no trinômio: produtividade, qualidade e flexibilidade. Ou seja, a competitividade da organização está fundamentada na sua capacidade de perceber mudanças e se preparar para enfrentá-las.

Segundo Montana e Charnov (1998), mesmo um autoconhecimento parcial das organizações permite uma postura pró-ativa em vez de meras reações. De acordo com Batocchio e Biagio (1999), conseguir identificar uma ameaça potencial antes que ela se torne um problema concreto pode significar uma vantagem competitiva considerável. Assim, faz-se necessário identificar as oportunidades de negócio e o desempenho da organização à luz destes.

Este contexto, associado à crescente participação do setor de serviços na capitalização de recursos para as organizações, torna especialmente relevante diagnosticar o grau de satisfação do consumidor com o desempenho da organização e de seus serviços. A Figura 1 busca ilustrar um dos problemas existentes neste contexto: dado um serviço ou conjunto de serviços, classificá-lo em uma classe de satisfação do cliente.

\section{Revisão Bibliográfica}

Segundo Denton (1991), Grönross (1980) e Silva (1998), dentre outros autores, as abordagens usualmente adotadas para o tratamento deste problema efetuam perguntas objetivas aos consumidores.
As questões objetivas enfocam critérios ou indicadores específicos. As respostas a essas questões permitem avaliar o desempenho do serviço à luz de cada critério. Analisando-se essas respostas busca-se classificar a qualidade do serviço prestado pela organização. A construção e aplicação de modelos neste contexto desenvolvem-se nas seguintes etapas:

- Definição de critérios de avaliação, à luz dos quais o serviço deve ser avaliado.

- Determinação de escalas para obtenção dos julgamentos de valor. Tanto para identificação do grau de importância de cada critério, quanto para a avaliação do desempenho do serviço à luz de cada critério.

- Especificação de um mecanismo para a coleta dos julgamentos de valor.

- Definição do peso ou grau de importância associado a cada critério. A atribuição destes pesos é um julgamento subjetivo, mesmo quando definidos através de uma decisão consensual ou mesmo por votação.

- Avaliação da importância ou peso de cada critério considerado na modelagem. Nesta etapa, observam-se avaliações subjetivas, pelas características inerentes ao problema de julgamento da importância dos critérios.

- Julgamento do desempenho do serviço à luz dos critérios, atribuindo-lhe uma nota ou índice de desempenho em cada critério. Também neste momento observa-se que alguns desses critérios são avaliados de forma subjetiva, pelas características inerentes aos mesmos.

- Mensuração do desempenho do serviço sob um ponto de vista global. Em geral, é adotada a média ponderada, como indicador do desempenho global do serviço.

Figura 1: Problemas de classificação ordenada.

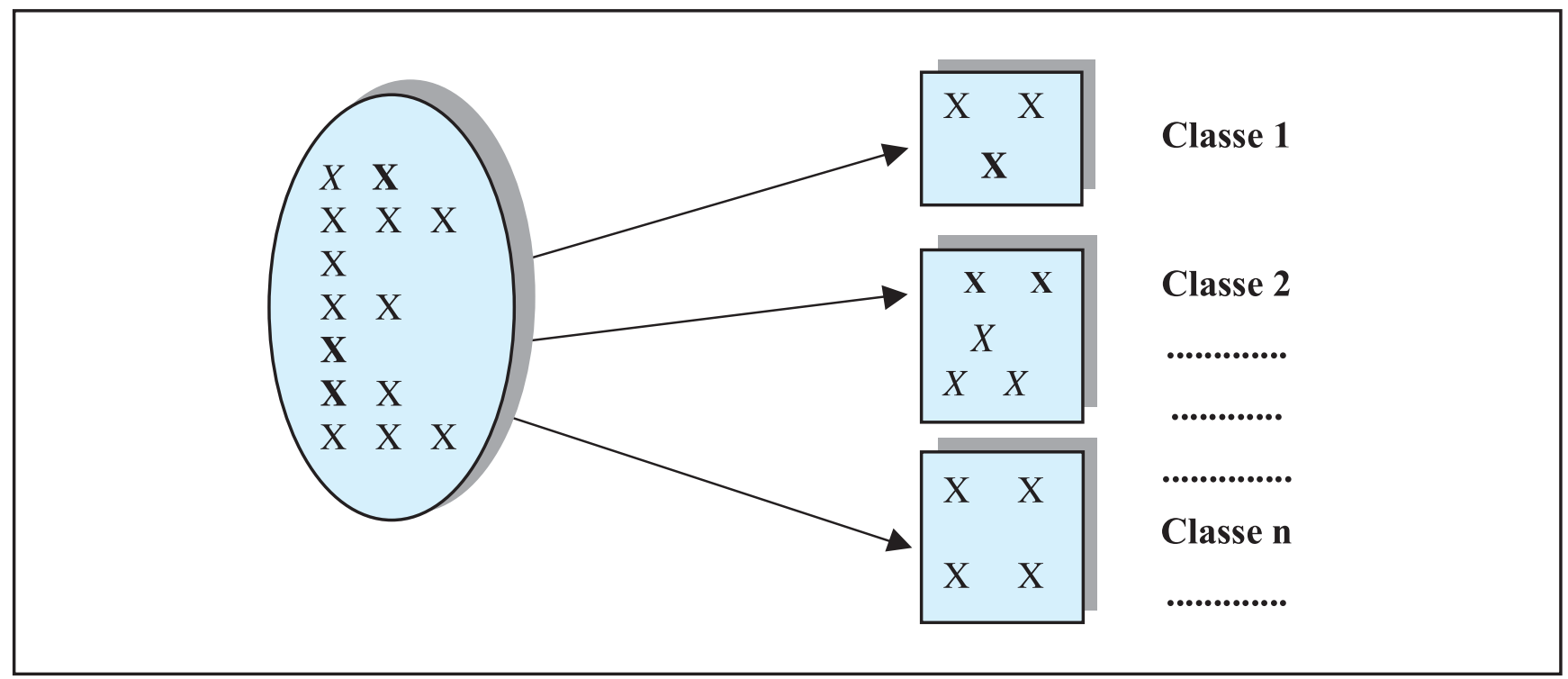

Fonte: Adaptado de MOUSSEAU et al. (1999). 
Assim, a resolução do problema em foco envolve a análise do desempenho do serviço à luz de múltiplos critérios ou fatores. Observa-se que esta análise envolve, mesmo para as questões objetivas, julgamentos subjetivos buscando a mensuração de propriedades não-tradicionalmente mensuráveis como: cortesia; conforto; e, satisfação (alguns autores denominam tais variáveis por variáveis não mensuráveis).

Os modelos usualmente construídos para a solução de problemas desta categoria não utilizam abordagens desenvolvidas especificamente para tratar de problemas com estas características (múltiplos critérios e variáveis subjetivas). Tais abordagens estão enquadradas na categoria do Auxílio Multicritério à Decisão (AMD) ou MCDA (Multicriteria Decision Aid).

- Grigoroudis et al. (2001) apresentam o método MUSA (Multicriteria Satisfaction Analysis), baseado em um sistema de desagregação de preferências construído a partir dos princípios da análise de regressão ordinal. A idéia original que levou ao desenvolvimento do MUSA foi apresentada inicialmente em Siskos et al. (1998), tendo sido aplicada à avaliação de riscos de crédito financeiro.

- Mihelis et al. (1998) apresentam um estudo sobre a satisfação de clientes atendidos no Banco da Grécia. A modelagem deste estudo fundamenta-se no método MUSA.

- A aplicação do MUSA também é explorada por Politis e Siskos (2004) na avaliação da qualidade do Departamento de Engenharia de Produção e Gestão da Universidade Técnica de CRETA (Department of Production Engineering and Management of the Technical University of Crete). Os critérios considerados nesta modelagem tratam aspectos ligados a três diretrizes: operação e processos internos; satisfação dos estudantes; e aceitação pelo mercado de trabalho.

\section{torna especialmente relevante diagnosticar}

\section{o grau de satisfação do consumidor com o desempenho da organização e de seus serviços.}

No Brasil, algumas abordagens para avaliação da satisfação de clientes apoiadas em métodos desenvolvidos no âmbito do Auxílio Multicritério à Decisão (AMD) ou MCDA(Multicri-

Alguns trabalhos foram desenvolvidos buscando a integração de conceitos de Auxílio Multicritério à Decisão (Multicriteria Decision Making, MCDM) à avaliação do grau de satisfação de consumidores. Dentre os trabalhos realizados nesta direção citam-se aqui os seguintes:

- West e Huang (1995) apresentam uma modelagem multicritério para a atribuição de pesos a critérios em sistemas de qualidade. Esta abordagem utiliza uma planilha eletrônica para implementar a comparação paritária da importância dos critérios. As comparações paritárias são agregadas em uma função utilidade e depois normalizadas obtendo-se a importância de cada critério. Os critérios são definidos com base na ISO 9000. É importante ressaltar que, apesar de usar julgamentos paritários, a escala adotada não considera a reciprocidade de julgamentos.

- Tsaur et al. (2002) propõem uma abordagem que integra os conceitos do Auxílio Multicritério à Decisão a conceitos de lógica nebulosa na avaliação da qualidade de serviços prestados por linhas aéreas. Neste trabalho: a atribuição de pesos aos critérios é feita com apoio do Método de Análise Hierárquica (Analytic Hierarquic Process, AHP, Saaty (1980)); e a avaliação do serviço baseia-se no método de Ordenação de Preferências por Similaridade (TOPSIS, Order Preference by Similarity to Ideal Solution - Hwang e Yoon (1981). teria Decision Aid) também têm sido propostas.

- Corrêa (2004) propõe o emprego do Método de Análise Hierárquica para gerar os pesos em um sistema de avaliação do grau de satisfação de usuários de imóveis com o desempenho do mesmo.

- Freitas (1997) propõe uma adaptação inédita do método ELECTRE III para classificar a qualidade dos serviços prestados por organizações prestadoras de serviços. Neste trabalho, investiga-se a aplicação desta abordagem à classificação dos "serviços de oficina de manutenção de automóveis de passeio" prestados por uma concessionária de automóveis. Um extrato desta pesquisa, destacando os principais aspectos da mesma, está publicado em Freitas e Costa (1998). Esta abordagem é também explorada em Mansur (2000) e Mansur e Costa (2001), que experimentam a aplicação desta adaptação do método ELECTRE III à avaliação do grau de satisfação de consumidores dos serviços oferecidos por uma pequena empresa do setor gastronômico. Este trabalho propõe um pré-processamento estatístico dos julgamentos, para eliminar os "pontos fora da curva" no conjunto de julgamentos.

O ELECTRE III foca problemas de ordenação. Ou seja: estabelecer uma posição relativa entre alternativas. Abordagens foram desenvolvidas para a adoção do ELECTRE 
III como método para a classificação ordenada, dentre as quais o MEMSOR (BANA; COSTA, 1990) e o ELEQUAL (FREITAS,1997; FREITAS; COSTA, 1998). No entanto, a utilização do ELCTRE III em problemas de classificação ordenada não é imediata, implicando em abstrações e adaptações que "forçam" o método. Percebendo este fato, Costa e Freitas (2000) propõem a aplicação do ELECTRE TRI (Elimination Et Choix Traduisant la Realité) reportado em YU (1992), para a classificação do grau de satisfação de clientes de serviços. Esta nova proposta baseia-se no fato do ELECTRE TRI ter sido desenvolvido para tratar de problemas de classificação ordenada. Experimentações a respeito desta abordagem são exploradas em Barroso (2002), Ribeiro (2003) e Ribeiro e Costa (2005), que investigaram a aplicação da mesma no âmbito dos serviços prestados por uma Universidade.

\section{Justificativa e objetivo}

Apesar dos avanços obtidos na aplicação da análise multicritério à avaliação da satisfação de consumidores, ainda existem aspectos a serem explorados neste contexto. Utilizando os dados coletados em Mansur (2000), o presente trabalho investiga a aplicação do Método ELECTRE TRI à avaliação do grau de satisfação de consumidores dos serviços oferecidos por uma pequena empresa do setor gastronômico.

Mais especificamente, este trabalho considera o préprocessamento estatístico dos julgamentos proposto em Mansur (2000); e propõe uma forma inédita para se especificar os limites de preferência e de indiferença utilizados no ELECTRE TRI. Vale destacar que, na revisão bibliográfica efetuada para a elaboração do presente trabalho, não se identificou que a forma de definição destes limites venha sendo elaborada de forma sistemática.

\section{BASE CONCEITUAL: MÉTODO ELECTRE TRI}

A abordagem apresentada neste artigo fundamenta-se nos conceitos do Auxílio Multicritério à Decisão (AMD). Mais especificamente, busca-se uma adaptação do ELECTRE TRI. Assim, para facilitar o entendimento daqueles que não estão familiarizados com este método ou com o AMD, apresenta-se aqui uma breve discussão sobre o AMD e o ELECTRE TRI.

Conforme reportado em Arrow (1963), Saaty (1980), Chankong (1983), Zeleny (1982), Roy e Boyssou (1985), a tomada de decisão em um ambiente complexo envolve a consideração de múltiplos critérios. Segundo reportado em Bana e Costa (1988) e corroborado por Vincke (1992), as abordagens de AMD podem ser classificadas como: abordagens do critério único de síntese; abordagens interativas local; e abordagens de subordinação.
- As abordagens do critério único de síntese caracterizamse por buscar uma função que agregue diferentes funções de utilidade em uma função única. Os métodos que se baseiam nesta abordagem têm sido classificados como pertencentes à Escola Americana de métodos multicritério. Dentre os métodos e teorias que se baseiam nesta abordagem, são aqui destacados: o AHP (SAATY, 1980), a Teoria da Escolha Social (ARROW, 1963) e a Teoria da Utilidade Multiatributo (Multi-Attribute Utility Theory, MAUT), apresentada em Fishburn (1970) e consolidada em Keeney e Raiffa (1976);

- Segundo ANTUNES et al. (1989), as abordagens interativas alternam fases de cálculo com fases de decisão, nas quais o analista de decisão interage com o modelo, construindo a decisão mais adequada. Este tipo de abordagem é especialmente importante em situações em que se esteja buscando uma única solução que seja ótima ou que esteja próxima do ponto ótimo.

- No âmbito dos Métodos de Subordinação, um conjunto finito de alternativas/ações (A) são valoradas sobre uma família/vetor de critérios (F), construindo-se relações de subordinação não compensatórias entre as alternativas. Buscando estabelecer uma ilustração didática deste conceito, apresenta-se a seguinte reflexão:

"Para se estabelecer bem a diferença entre o emprego da média ponderada e dos métodos de superação, pode-se tomar uma analogia com o que acontece em uma partida de voleibol no confronto entre os times A e B. Se no primeiro set $\mathrm{B}$ ganha de A por 25 a Zero, porém nos demais três sets A ganha de B por 25 a 20, duas análises são feitas:

1) Usar a média ponderada para obter o resultado final. Neste caso B seria o vencedor da partida por 85 a 75 .

2)Usar o número de sets para definir o vencedor. Neste caso, A seria o vencedor por 3 a 1 .

O princípio fundamental dos métodos de superação pode ser considerado semelhante a esta segunda abordagem, se considerarmos que cada set equivale a cada um dos critérios da análise multicritério."

A mais conhecida família de métodos de Subordinação é a família ELECTRE. Estes métodos têm origem no trabalho de Roy (1968), sendo adotados tanto no apoio quanto na tomada de decisão. A família ELECTRE é composta, atualmente, pelos seguintes métodos: ELECTRE (ROY, 1968), ELECTRE II (ROY; BERTIER, 1971), ELECTRE III (ROY, 1978), ELECTRE IV (ROY; HUGONNARD, 1981), ELECTRE IS (ROY; M.,1985) e ELECTRE TRI (MOUSSEAU et al., 1999; YU,1992). A seguir apresenta-se um breve resumo a respeito de cada um destes métodos:

- Métodos ELECTRE I e IS. Particionam o conjunto de alternativas em dois subconjuntos: alternativas não domi- 
nadas; e alternativas dominadas. O método ELECTRE I usa o conceito de critério verdadeiro, segundo o qual há uma concordância plena (em um critério genérico j) de que uma alternativa $a$ é pelo menos tão boa quanto uma outra alternativa $b$ se o desempenho de $b$ for inferior ao de $a$ (mesmo que apenas infinitesimalmente inferior). O ELECTRE IS usa o conceito de pseudocritério, segundo o qual há uma concordância plena (em um critério genérico j) de que uma alternativa $a$ é pelo menos tão boa quanto uma outra alternativa $b$ mesmo que o desempenho de $a$ seja um pouco menor
Portanto, cada um dos métodos ELECTRE tem uma finalidade distinta. Ou seja: a escolha do método depende do tipo de problema que se deseja resolver. A seguir apresenta-se uma breve descrição do Método ELECTRE TRI, adotado no presente trabalho, devido às características específicas do problema abordado.

\section{O Método ELECTRE TRI}

O Método ELECTRE TRI caracteriza-se por ter sido desenvolvido especificamente para tratar de problemas específicos de classificação ordenada. Ou seja: dado um conjunto de $\mathrm{A}=\left[\mathrm{A}_{1}, \mathrm{~A}_{2}, \ldots . . \mathrm{A}_{\mathrm{n}}\right]$ alternativas, o ELECTRE TRI associa-as a um conjunto de k-classes ordenadas $\mathrm{C}=\left[\mathrm{C}_{1}, \mathrm{C}_{2}, \ldots \ldots \mathrm{C}_{\mathrm{k}}\right]$. A Figura 2 ilustra um conjunto formado por $\mathrm{h}+1$ classes, delimitadas por $\mathrm{h}$ limites de classes, considerando um conjunto de critérios $F=\left[g_{1}, g_{2}, \ldots, g_{m}\right]$. Uma classe genérica $\mathrm{C}_{\mathrm{h}}$ é delimitada por um limite inferior $b_{h}$ e um limite superior $b_{h}-1$.

Este método integra funções que dão suporte ao decisor no processo de preferência e reduzem o esforço cognitivo requerido na fase de modelagem. Este método classifica as alternativas através: da construção de uma relação de subor-

(dentro de um limite aceitável $q$ ) do que o de $b$. Ou seja: o pseudocritério considera a possibilidade de hesitação ou incerteza de um avaliador ao afirmar que uma alternativa é, de fato, pelo menos tão boa quanto uma outra.

- Métodos ELECTRE II, III e IV. Ordenam as alternativas presentes no conjunto de alternativas viáveis. O método ELECTRE II usa o conceito de critério verdadeiro para estabelecer as relações de subordinação, porém usa uma estrutura de relaxamento para obter a ordenação das alternativas. Nesta estrutura de relaxamento considera-se a construção de dois grafos: Grafo Forte; e Grafo Fraco. Os métodos ELECTRE III e IV utilizam o conceito de pseudo-critério para estabelecer uma relação de credibilidade, a partir do qual ordenam as alternativas através de um processo de "destilação". O método ELECTRE IV é utilizado em problemas de ordenação quando não se pode (ou não se deseja) atribuir pesos aos critérios.

- Método ELECTRE TRI. O método ELECTRE TRI busca resolver problemas de classificação ordenada. Neste tipo de problema busca-se classificar alternativas presentes no conjunto de alternativas viáveis em classes que mantêm uma relação de preferência entre si. Este método usa o conceito de pseudocritério para estabelecer as relações de subordinação. dinação $\mathrm{S}$, que caracteriza como as alternativas são comparadas aos limites das classes; e da exploração (através de procedimentos de classificação) da relação $\mathrm{S}$.

\section{Relação de subordinação no ELECTRE TRI}

A relação de subordinação é construída para tornar possível a comparação de uma alternativa $a$ com um limite padrão $b_{h}$. A afirmação de que $\mathrm{aSb}_{\mathrm{h}}$ significa que " $a$ não tem um desempenho pior do que o limite $b_{h}$ ". Na validação da afirmação $\mathrm{aSb}_{\mathrm{h}}$ devem-se verificar duas condições:

- Concordância: para que $\mathrm{aSb}_{\mathrm{h}}\left(\right.$ ou $\mathrm{b}_{\mathrm{h}} \mathrm{Sa}$ ) seja aceita, uma maioria suficiente de critérios deve ser a favor desta afirmação.

- Não-discordância: quando na condição de concordância esperada, nenhum dos critérios na minoria deve se opor à afirmação $\mathrm{aSb}_{\mathrm{h}}(\mathrm{ou} \mathrm{b} \mathrm{ha})$.

Os seguintes passos são seguidos na obtenção desta relação:

- Computar o índice de concordância parcial $c_{j}\left(a, b_{h}\right)$ e $c_{j}\left(b_{h}, a\right)$,

- Computar o índice de concordância geral c $\left(a, b_{h}\right)$,

- Computar o índice de discordância parcial $\mathrm{d}_{\mathrm{j}}\left(\mathrm{a}, \mathrm{b}_{\mathrm{h}}\right)$ e $\operatorname{dj}\left(b_{h}, a\right)$

- Computar a relação de subordinação fuzzy, conforme o índice de credibilidade $\sigma\left(a, b_{h}\right)$, 
- Determinar um corte $\lambda$ da relação fuzzy para obter uma relação de subordinação. Isto é: $\operatorname{Se} \sigma\left(a, b_{h}\right) \geq \lambda \Rightarrow a b_{h}$.

O grau de credibilidade da relação de subordinação $\sigma\left(a, b_{h}\right)$ expressa com que intensidade se pode "acreditar" que "a subordina $\mathrm{b}_{\mathrm{h}}$ " de acordo com o índice de concordância global $c_{j}\left(a, b_{h}\right)$ e com o índice de discordância d $d_{j}\left(a, b_{h}\right)$.

A tradução de uma relação de subordinação fuzzy obtida entre uma relação de subordinação $S$ é feita sobre o significado de um nível de corte $\lambda$. Este nível de corte $(\lambda)$ é considerado como o menor valor do índice de credibilidade compatível com a afirmação de que "a subordina $b_{\mathrm{h}}$ ". Isto é: se $\sigma\left(a, b_{h}\right) \geq \lambda$, então $a b_{h}$.

\section{Procedimentos de classificação no ELECTRE TRI}

A regra do procedimento de exploração é realizada para analisar o modo em que uma alternativa $a$ é comparada com os limites padrão determinados para a classe na qual $a$ deve ser enquadrada. Dois procedimentos de classificação são avaliados.

- O procedimento de classificação descendente é descrito a seguir:

- Compare $a$ sucessivamente com $\mathrm{b}_{\mathrm{i}}$, para $\mathrm{i}=\mathrm{p}, \mathrm{p}-1$, ..., 1.

- Encontre um $b_{h}$ que seja o primeiro limite tal que $\mathrm{aSb}_{\mathrm{h}}$.
- Classifique $a$ na classe $\mathrm{C}_{\mathrm{h}+1}$. Ou seja, na classe limitada inferiormente pelo limite $b_{h}$ ).

- O procedimento de classificação ascendente é descrito a seguir:

- Compare $a$ sucessivamente com bi, i=1,2, .., p.

- Encontre o primeiro $b_{h}$ para o qual $b_{h}>a$.

- Classifique $a$ na classe limitada superiormente por este limite. Ou seja, classifique $a$ na classe $\mathrm{C}_{\mathrm{h}}$.

Comparação entre os dois procedimentos de classificação.

Sendo estes dois procedimentos diferentes, conseqüentemente, pode ocorrer a classificação de algumas alternativas em diferentes classes, pois: o procedimento otimista tende a classificar as alternativas em classes mais altas; o procedimento descendente tende a classificar as alternativas nas categorias mais baixas; e o procedimento ascendente tende a classificar as alternativas nas categorias mais elevadas.

No caso do ELECTRE TRI, uma divergência entre essas classificações indica uma incapacidade do sistema em comparar o elemento sendo classificado a pelo menos um dos perfis das classes de equivalência utilizadas. Tradicionalmente, considera-se que esta incapacidade pode ser causada tanto por incoerência do avaliador, quanto pelo modelo de classificação (incluindo o conjunto de critérios) ou pelo sistema de coleta de dados. Costa (2005) considera que a divergência das classificações é comum em situações em que

Figura 2: Classes de referência.

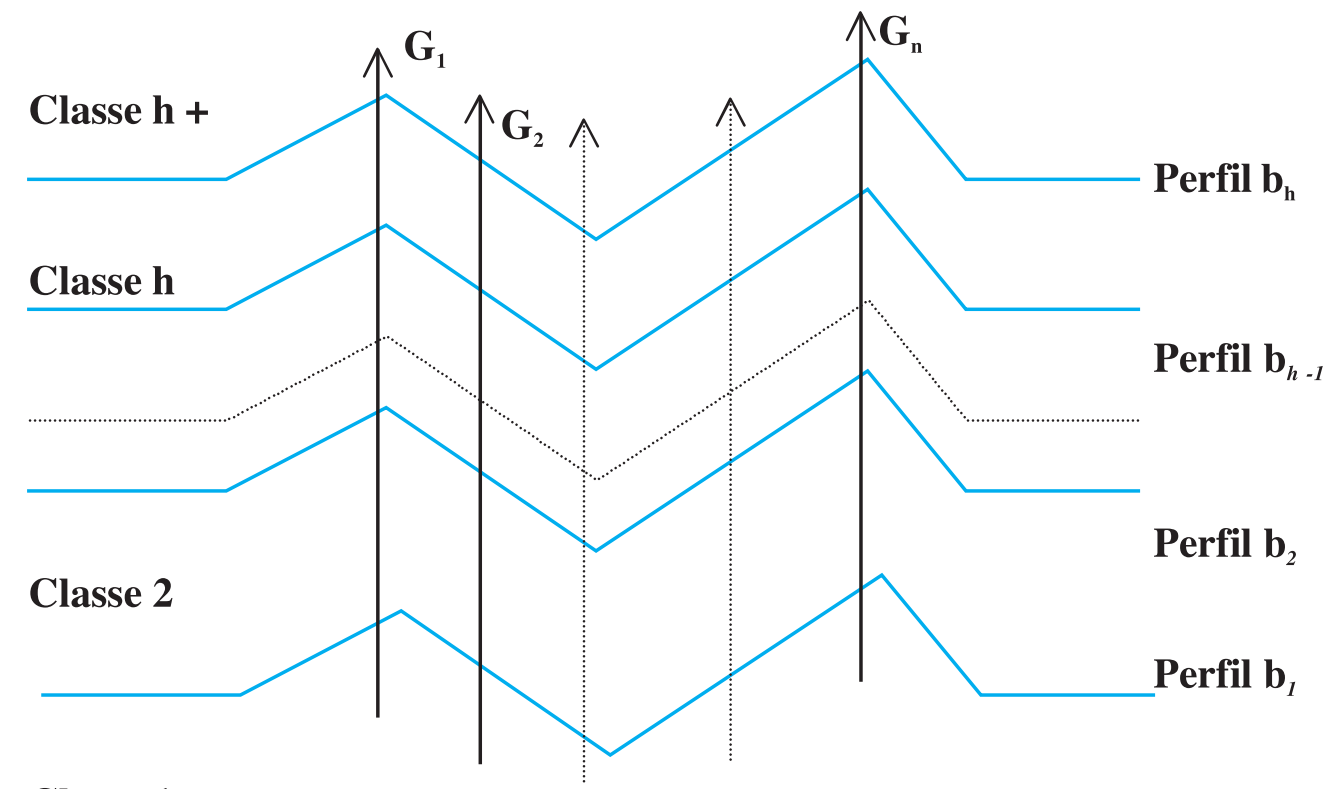

\section{Classe 1}


haja critérios conflitantes (por exemplo: custo e qualidade), sendo neste caso inerente ao problema e não devendo ser considerada com uma falha na modelagem.

Assim, quando ocorre divergência entre as classificações pessimista e otimista, o classificador deve adotar uma das duas classificações de acordo com o seu perfil (mais exigente ou menos exigente).
- Importância ou influência de cada FCS para a satisfação do consumidor. Esta influência é aqui denotada por $\mathrm{GI}_{\mathrm{j}}$. (Grau de Importância do critério $j$ ).

- Grau de satisfação do consumidor com o serviço à luz de cada FCS. Este grau de satisfação é aqui denotado por $\mathrm{GS}_{\mathrm{j}}$.(Grau de Satisfação à luz do critério $j$ ).

Neste processo os consumidores emitem julgamentos de valor com base nas escalas definidas nos passos (c) e (d).

\section{s modelos usualmente construídos para} a solução de problemas desta categoria não utilizam metodologias desenvolvidas especificamente para tratar de problemas com estas características (múltiplos critérios e variáveis subjetivas].

\section{ABORDAGEM PROPOSTA}

Neta seção, descrevem-se as etapas da abordagem aqui proposta:

a. Identificar os serviços a serem avaliados. Nesta etapa, busca-se identificar o objeto de estudo, ou seja, os serviços que serão avaliados, sob a ótica da satisfação do consumidor.

b. Especificar os critérios ou FCS (Fatores Críticos de Satisfação). Estabelecer e listar os FCS a serem considerados na avaliação do grau de satisfação dos consumidores. Conforme reportado em Azevedo (2001), a percepção destes FCS encontra-se num contexto pessoal e ambiental, sendo um processo em permanente evolução dada a dinâmica do ambiente e da própria percepção pessoal de quem define os FCS. Assim, FCS podem ser diferentemente percebidos por diferentes clientes, portanto, devem ser definidos buscando-se atender ao público alvo do serviço.

c. Especificar a escala para os julgamentos da importância (peso) de cada FCS. O peso está associado à percepção do cliente quanto à importância ou influência do FCS no grau de satisfação do cliente com o serviço. Assim, é importante utilizar uma escala que permite captar esta percepção.

d. Especificar a escala de julgamentos dos desempenhos de cada organização à luz de cada FCS. Esta escala deve permitir ao avaliador a emissão de seus julgamentos de valor sobre o desempenho do serviço. Pode ser construída uma escala para cada critério.

e. Coletar julgamentos de valor à luz de cada FCS. Nesta etapa, busca-se captar a percepção dos consumidores quanto a: f. Análise dos dados obtidos na pesquisa. Nesta etapa, os valores de $\mathrm{GS}_{\mathrm{j}}$ e $\mathrm{GI}_{\mathrm{j}}$, emitidos por cada consumidor em cada critério, são analisados sob a óptica da estatística descritiva. Ainda no âmbito do tratamento dos dados, sugere-se identificar e eliminar "pontos fora da curva". Nesta etapa deve-se identificar e eliminar dados que possam estar contaminando a amostra. Para tal, sugere-se o Critério de Chauvenet, reportado em Dally e Riley (1998) e de grande utilização no âmbito da metrologia para o tratamento de dados amostrais associados a experimentos de medição. Este procedimento estatístico busca determinar, a partir de um conjunto de medidas, quais destas são resultantes de um erro de medição ou de registro e estão contribuindo, desnecessariamente, para aumentar o desvio padrão. Com o emprego deste método busca-se eliminar os "pontos fora da curva" que porventura existam na amostra. A aplicação do Critério de Chauvenet está estruturada na execução das seguintes etapas:

- Cálculo do Desvio relativo máximo $\left(\mathrm{DR}_{\max }\right)$ :

$\mathrm{DR}_{\max }=\left(\mathrm{X}_{\max }-\mathrm{X}_{\mathrm{m}}\right) / \mathrm{S}_{\mathrm{x}}$

onde:

$\mathrm{X}_{\max }$ : Maior valor do conjunto de medidas.

$\mathrm{X}_{\mathrm{m}}$ : Média do conjunto de medidas

$\mathrm{S}_{\mathrm{x}}$ : Desvio Padrão associado ao conjunto de medidas.

- Cálculo do Desvio relativo mínimo $\left(\mathrm{DR}_{\mathrm{min}}\right)$ :

$\mathrm{DR}_{\min }=\left|\mathrm{X}_{\min }-\mathrm{X}_{\mathrm{m}}\right| / \mathrm{S}_{\mathrm{x}}$

onde:

$\mathrm{X}_{\min }$ : Menor valor do conjunto de medidas.

$\mathrm{X}_{\mathrm{m}}$ : Média do conjunto de medidas

$\mathrm{S}_{\mathrm{x}}$ : Desvio Padrão associado ao conjunto de medidas.

Os valores obtidos para $\mathrm{DR}_{\text {max }}$ e $\mathrm{DR}_{\text {min }}$, são comparados a um Desvio Relativo Padrão $\left(\mathrm{DR}_{0}\right)$. Assim: 
$\mathrm{Se}, \mathrm{DR}_{\max }>\mathrm{DR}_{0}, \mathrm{X}_{\text {máx }}$ é eliminado, caso contrário o mesmo é mantido na amostra.

Se: $\mathrm{DR}_{\min }>\mathrm{DR}_{0}, \mathrm{X}_{\min }$ é eliminado, caso contrário o mesmo é mantido na amostra.

A Tabela 1 ilustra os diferentes valores assumidos por $\mathrm{DR}_{0}$, em função do tamanho da amostra.

g. Identificar as classes de equivalência juntamente com seus respectivos limites. Neste passo, estabelecem-se "classes-padrão" de satisfação. Estas classes são definidas por limites superiores e inferiores, também denotados por "perfis".

h. Estabelecer os limites de preferência $\left(p_{j}\right)$ e de indiferença $\left(q_{j}\right)$ para cada critério $\boldsymbol{j}$. Estes limites permitem considerar a natureza imprecisa, intrínseca às avaliações por julgamento de valor. Não existe, na literatura, um consenso para a determinação destes limites quando se considera avaliação conjunta por múltiplos avaliadores os métodos ELECTRE foram desenvolvidos para tratar de decisões que envolvam uma única unidade de decisão. Considerando que os valores $\mathrm{p}_{\mathrm{j}}$ e $\mathrm{q}_{\mathrm{j}}$ estão associados à dispersão do julgamento pela amostra dos consumidores votantes e adotando uma escala que possui intervalo constante I, no presente trabalho sugere-se:

$\mathrm{p}_{\mathrm{j}}=\mathrm{q}_{\mathrm{j}}=\min$ (Coeficiente de variação da amostra associada aos $\left.\mathrm{GS}_{\mathrm{j}} ; \mathrm{I} / 2\right)$

i. Executar o algoritmo de classificação do ELECTRE TRI. Nesta etapa obtém-se a Classificação da Satisfação do consumidor com o serviço, em uma das classespadrão. Para isto utiliza-se o método de subordinação (ELECTRE TRI), que se baseia em duas estratégias de classificação:

- Classificação pessimista (mais exigente): um serviço $\mathrm{X}_{\mathrm{j}}$ é classificado em uma classe genérica $\mathrm{h}$, se existem argumentos suficientes (com um grau de credibilidade mínimo o) para afirmar que o desempenho do serviço $h$ subordina o perfil h-1. Ou seja, um serviço $X_{j}$ é classifi- cado em uma classe genérica $\mathrm{h}$ se tem um desempenho pelo menos tão bom quanto o perfil h-1 em um número significativo de critérios.

- Classificação otimista (menos exigente): um serviço $\mathrm{X}_{\mathrm{j}}$ é classificado em uma classe genérica $\mathrm{h}$ se existem argumentos suficientes (com um grau de credibilidade mínimo o) para afirmar que perfil h subordina o desempenho do serviço $\mathrm{X}_{\mathrm{j}}$. Ou seja, um serviço é classificado em uma classe genérica $h$ se tem um desempenho imediatamente inferior ao perfil h em um número significativo de critérios.

O grau de credibilidade é uma medida da intensidade com que se pode "acreditar" na classificação obtida, sendo definido a partir de uma integração entre o conceito de concordância (o quanto o consumidor "concorda" com a classificação) e o conceito de discordância (o quanto o consumidor rejeita a classificação).

Quando ocorre divergência entre as classificações pessimista e otimista, o classificador deve adotar uma das duas classificações de acordo com o seu perfil, (mais exigente ou menos exigente). Este procedimento também é adotado em outras situações de decisão em que se classifica o perfil do decisor em otimista ou pessimista, como na Teoria dos Jogos. Mais ainda, no caso do ELECTRE TRI, uma divergência entre estas classificações indica uma incapacidade do sistema em comparar o elemento sendo classificado a pelo menos um dos perfis das classes de equivalência utilizadas. Esta incapacidade pode ser causada pelo avaliador, pelo modelo de classificação (incluindo o conjunto de critérios) ou pelo sistema de coleta de dados (incluindo as escalas utilizadas). A identificação de incomparabilidades constitui-se em um sinal adicional sobre inconsistências na construção do modelo não detectadas pelos métodos convencionais.

\section{APLICAC̣̃̃O DA ABORDAGEM PROPOSTA.}

Objetivando ilustrar a aplicação da abordagem proposta, apresenta-se aqui um caso de aplicação da mesma.

Tabela 1: Valores para $\mathbf{D R}_{0}$.

\begin{tabular}{|c|c|c|c|}
\hline $\begin{array}{c}\text { No DE MEDIÇÕES } \\
(\mathbf{n})\end{array}$ & $\begin{array}{c}\text { DESVIO RELATIVO PADRÃO } \\
\left(\mathbf{D R}_{\mathbf{0}}\right)\end{array}$ & $\begin{array}{c}\text { № DE MEDIÇÕES } \\
(\mathbf{n})\end{array}$ & $\begin{array}{c}\text { DESVIO RELATIVO } \\
\text { PADRÃO (DR }^{\text {D }}\end{array}$ \\
\hline 4 & 1,54 & 25 & 2,33 \\
\hline 5 & 1,65 & 50 & 2,57 \\
\hline 6 & 1,80 & 100 & 2,81 \\
\hline 10 & 1,96 & 300 & 3,14 \\
\hline 15 & 2,13 & 500 & 3,29 \\
\hline
\end{tabular}

Fonte: Dally e Riley (1998). 
a. Identificar o objeto de estudo. Foi considerada uma microempresa do setor de varejo alimentício (bombonnière) denominada Delícias ${ }^{\circledR}$, localizada no Parquecentro Shopping, na cidade de Campos dos Goytacazes. Essa loja varejista serviu de laboratório para este estudo de caso.

b. Especificar os critérios ou FCS. Foram considerados os FCS apresentados a seguir, os quais foram adaptados de Freitas (1997), com base na revisão bibliográfica e em discussão com o "dono do negócio". Assim, a modelagem aqui apresentada busca captar a percepção da satisfação do cliente nos critérios em que o "dono do negócio" julgava relevantes, considerando-se peculiaridades do negócio. Os seguintes fatores foram considerados:

- Cortesia - Educação e cordialidade dos vendedores no atendimento.

- Receptividade - Dedicação e interesse dos vendedores na solução de problemas apresentados pelos clientes.

- Rapidez - Rapidez e Agilidade no atendimento de modo que o cliente não tenha a sensação de demora no atendimento.

- Instalações - Organização e disposição dos móveis, assim como o conforto do ambiente para o cliente.

- Acesso - Facilidade de Acesso: Estacionamento do Shopping e visibilidade da loja.

- Limpeza - Aspecto de higiene do local e dos atendentes.

- Preço - Preço praticado na venda dos produtos.

- Variedade - Diversidade de produtos disponíveis para consumo na loja.

- Novidade - Grau de inovação e originalidade da linha de produtos comercializados.

c. Especificar a escala para os julgamentos dos graus de importância (pesos) de cada FCS. Com base nas escalas utilizadas em Bana e Costa (1990) e Herrera e Costa (2001), que trabalharam com problemas de classificação com características semelhantes às do problema em foco, adotou-se escala ilustrada no Tabela 2. Com base nessa escala, o avaliador pode julgar a importância do critério utilizando uma linguagem verbal.

Tabela 2: Escala para julgamento da importância dos critérios.

\begin{tabular}{|c|c|}
\hline ESCALA VERBAL & VALOR NUMÉRICO \\
\hline Altíssima & 4 \\
Alta & 3 \\
Média & 2 \\
Baixa & 1 \\
Desprezível & 0 \\
\hline
\end{tabular}

d. Especificar a escala de julgamentos do serviço à luz de cada FCS. A escala de julgamentos utilizada para a avaliação da satisfação do consumidor com o serviço prestado é aquela apresentada na Tabela 3. Esta escala está fundamentada nos conceitos reportados nos trabalhos de Likert (1932), Miller (1954) e Parducci (1965) e busca possibilitar ao avaliador "contar nos dedos" no ato de emissão de sua opinião.

Tabela 3: Escala para avaliação do nível de satisfação.

\begin{tabular}{|c|c|}
\hline ESCALA VERBAL & VALOR NUMÉRICO \\
\hline Muito Bom & 5 \\
Bom & 4 \\
Médio & 3 \\
Ruim & 2 \\
Muito Ruim & 1 \\
\hline
\end{tabular}

e. Coletar julgamentos de valor à luz de cada FCS. Com o auxílio de um formulário, as opiniões dos consumidores foram coletadas, com o consumidor externando a sua percepção quanto ao $\mathrm{GI}_{\mathrm{j}}$ e ao $\mathrm{GS}_{\mathrm{j}}$. A coleta das opiniões foi efetuada com a aplicação de um questionário. Os questionários preenchidos pelos clientes estão disponíveis para consulta em Mansur (2001). Foram considerados como válidos, um total de 72 questionários. Uma das principais dificuldades encontradas na coleta dos dados foi a falta de interesse dos respondentes em preencher o questionário. Devido a particularidade do serviço (bombonnière) os seguintes fatores amplificaram estas dificuldades:

- Alguns dos consumidores eram crianças, aos quais não se aplicava o questionário, pois os mesmos tinham dificuldade de entender "o que" era o questionário e "por que" deveriam respondê-lo.

- Alguns consumidores eram acompanhados por criança(s) que após efetuar(em) a compra ficava(m) ansiosa(s) em sair da loja para desenvolver outra atividade no shoppingprincipalmente para se divertir nas máquinas eletrônicas situadas próximo à loja.

- Por outro lado, alguns consumidores acompanhados por criança(s) após efetuarem a compra ficavam ansiosos para deixar a loja e desenvolver outra atividade no shopping antes que sua companhia gastasse em demasia na loja.

f. Análise dos dados obtidos na pesquisa. $\mathrm{O}$ desenvolvimento desta etapa foi estruturado em:

- Análise do comportamento da amostra. Para avaliar o comportamento da amostra à luz da estatística, foram considerados três parâmetros: coeficiente de variação; assimetria; e curtose.

Observou-se que os valores dos coeficientes de CV refletem um grau de homogeneidade aceitável para os dados obtidos. No entanto, o valor do coeficiente de variação 
igual a 0,296 para o grau de satisfação do critério preço bem superior aos obtidos para os demais critérios - reflete uma menor homogeneidade ou concordância dos clientes quanto à importância do critério preço. Isto implica em uma maior necessidade de análise no momento da adoção de alguma medida gerencial para melhoria do valor do GS para este critério.

Os resultados para o Coeficiente de Assimetria (As) indicam uma predominância da assimetria negativa com tendência à simetria. Este é um dos pressupostos (juntamente com um $\mathrm{CV}$ e um $\mathrm{K}$ dentro dos limites aceitáveis) para que possam ser desenvolvidos estudos de inferência estatística, servindo de suporte para tomada de decisões à luz de dados probabilísticos.

Observou-se, também, uma predominância leptocúrtica com respeito à Curtose. Apesar destes resultados não serem muito satisfatórios para fins de previsões estatísticas, é interessante notar que curvas leptocúrticas, caracterizam uma concentração de pontos em torno da média, o que, sob o ponto de vista de marketing, é bastante interessante por representar uma homogeneidade ou comportamento padrão dos consumidores.

- Aplicação do Critério de Chauvenet. Conforme poder ser observado do Quadro 1, para uma amostra com 94 elementos, tamanho da amostra deste estudo de caso, considera-se $\mathrm{DR}_{0}=2,57$. O Quadro 1 ilustra os valores de $\mathrm{DR}_{\text {máx }}, \mathrm{DR}_{\text {min }}$ e também a aplicação do Critério de Chauvenet a todas as amostras ou critérios. Empregando-se o Critério de Chauvenet, observa-se que não foram detectados "pontos fora da curva" nas amostras.

g. Estabelecer os limites de preferência $\left(p_{\mathrm{j}}\right)$ e de indiferença $\left(q_{j}\right)$ para cada critério $\boldsymbol{j}$. Conforme descrito na seção anterior, considerando que os valores $\mathrm{p}_{\mathrm{j}}$ e $\mathrm{q}_{\mathrm{j}}$ estão associados à dispersão do julgamento pela amostra dos consumidores votantes e que a escala adotada possui intervalo unitário, adotaram-se os valores apresentados no Quadro 2, para $\mathrm{p}_{\mathrm{j}}$ e $\mathrm{q}_{\mathrm{j}}$.

h. Identificar as classes de equivalência juntamente com seus respectivos limites. Conforme descrito na seção anterior, estas classes são definidas por limites superiores e inferiores, também denotados por "perfis". O Quadro 3 apresenta os valores dos limites adotados para cada classe, os quais foram definidos em função da escala apresentada na Tabela 3. A Figura 3 busca ilustrar as classes limitadas pelos limites superiores e inferiores.

i. Executar o algoritmo de classificação do ELECTRE TRI considerando os FCS Internos. Os julgamentos emitidos por cada julgador foram processados através do algoritmo de ordenação do

Quadro 1: Aplicação do Critério de Chauvenet.

\begin{tabular}{|l|l|c|c|c|}
\hline \multicolumn{1}{|c|}{ GRITÉRIO } & \multicolumn{1}{c|}{ MEDIDA } & DR $_{\text {MÁx }}$ & DR $_{\text {MIN }}$ & ALGUM PONTO EXCLUÍDO? \\
\hline \multirow{2}{*}{ Cortesia } & Satisfação & 0,360288 & $-7,165735$ & não \\
\cline { 2 - 5 } & Importância & 0,400616808 & $-11,18645396$ & não \\
\hline \multirow{2}{*}{ Receptividade } & Satisfação & 0,55331 & $-6,46801$ & não \\
\cline { 2 - 5 } & Importância & 0,513552591 & $-7,36092047$ & não \\
\hline \multirow{2}{*}{ Instalações } & Satisfação & 0,483141 & $-6,50382$ & não \\
\cline { 2 - 5 } & Importância & 0,517932397 & $-6,97216689$ & não \\
\hline \multirow{2}{*}{ Acesso } & Satisfação & 0,486343 & $-6,472097$ & não \\
\cline { 2 - 5 } & Importância & 0,38340579 & $-6,40835392$ & não \\
\hline \multirow{2}{*}{ Limpeza } & Satisfação & 0,64102 & $-5,69796$ & não \\
\cline { 2 - 5 } & Importância & 0,581675051 & $-6,32086889$ & não \\
\hline Preço & Satisfação & 0,269975 & $-9,66509$ & não \\
\cline { 2 - 5 } & Importância & 0,286972022 & $-14,79955711$ & não \\
\hline Variedade & Satisfação & 1,1159167 & $-2,478718$ & não \\
\cline { 2 - 5 } & Importância & 0,581879457 & $-3,57114255$ & não \\
\hline Novidades & Satisfação & 0,416747 & $-8,70265$ & Não \\
\cline { 2 - 5 } & Importância & 0,389490419 & $-9,95982643$ & Não \\
\cline { 2 - 5 } & Satisfação & 0,409101 & $-7,20019$ & Não \\
\cline { 2 - 5 } & Importância & 0,359937017 & $-8,00859862$ & Não \\
\hline
\end{tabular}


ELECTRE TRI, considerando um plano de corte (grau de credibilidade $=0,75$ ). O Anexo I apresenta um quadro que reporta os resultados obtidos classificação pessimista e classificação otimista para as avaliações de desempenho feitas por cada consumidor.

\section{sto significa que uma modelagem na qual se considere a média dos julgamentos} [como o "usuário Delícias"] pode mascarar significativamente o resultado quanto à identificação de incomparabilidades.

Considerando apenas os julgamentos para os quais não ocorreu incomparabilidade, observa-se que:

- Não houve indicação de classificação do serviço nas Classes "C"; "D"; e "E".

- Apenas o consumidor C54 classificou o serviço na Classe "B".

- Ocorreu uma preponderância da classificação do serviço na Classe "A".

No quadro do Anexo I, observa-se a ocorrência de incomparabilidades ao se considerar os julgamentos de 25 dos consumidores $(34,8 \%$ do total de consumidores entrevistados). Isto significa que para este conjunto de avaliadores o modelo deveria ser revisto. É relevante observar que este sinal não seria captado por um método de classificação baseado na média ponderada que considerasse os mesmos julgamentos. Tradicionalmente, considera-se que a incomparabilidade seja causada por: incoerência do avaliador; pelo modelo de classificação (incluindo o conjunto de critérios); ou pelo sistema de coleta de dados (incluindo as escalas utilizadas).

No entanto, a presença de incomparabilidades pode estar associada a características intrínsecas ao produto sob análise e à modelagem, não constituindo em falha do sistema de avaliação. Podem existir critérios que, mesmo sendo independentes, estejam alinhados e a sua avaliação seja convergente. Por outro lado, pode haver critérios que sejam conflitantes, o que induz divergências de desempenho. Por exemplo: critérios pertencentes à dimensão preço, em geral, são conflitantes com critérios pertencentes à dimensão qualidade ou prazo. Esta observação corresponde, por analogia, a situações destacadas na programação multiojetivo e na otimização por algoritmos genéticos nas quais não se encontra a um "Ótimo de Pareto".

Para o problema abordado nesta pesquisa, observa-se que o desempenho no critério preço é fortemente conflitante com o desempenho nos critérios, variedade, novidade, instalações, acesso, e limpeza. Em particular para os observadores cuja classificação indicou divergência pelos critérios pessimista e otimista. Ou seja, o fato de existir incomparabilidade não indica, necessariamente, uma falha do modelo pois pode indicar um comportamento não homogêneo da alternativa.

É interessante observar também o resultado da classificação do "usuário Delícias", para o qual não foi identificada incomparabilidade. Em cada critério, o julgamento deste "usuário" é a média dos julgamentos emitidos pelos demais usuários neste critério. Isto significa que uma modelagem na qual se considere a média dos julgamentos (como o "usuário Delícias") pode mascarar significativamente o resultado quanto à identificação de incomparabilidades.

Quadro 2: Valores adotados para $p_{\mathrm{j}}$ e $\mathrm{q}_{\mathrm{j}}$.

\begin{tabular}{|c|c|c|}
\hline CRITÉRIOS & GV $\left(G S_{j}\right)$ & $P_{j}=Q_{j}=\operatorname{MIN}\left(C V\left(G S_{j}\right) ; 0,50\right)$ \\
\hline Cortesia & 0,1105 & 0,1105 \\
\hline Receptividade & 0,1216 & 0,1216 \\
\hline Rapidez & 0,1212 & 0,1212 \\
\hline Instalações & 0,1218 & 0,1218 \\
\hline Acesso & 0,1373 & 0,1373 \\
\hline Limpeza & 0,0823 & 0,0823 \\
\hline Preço & 0,2961 & 0,2961 \\
\hline Variedade & 0,911 & 0,911 \\
\hline Novidades & 0,1099 & 0,1099 \\
\hline
\end{tabular}




\section{CONCLUSÕES}

Em geral, os julgamentos dependem da avaliação de diversas variáveis simultaneamente e de interpretações pessoais múltiplas, as quais variam de acordo com a experiência/preferência do avaliador. A abordagem proposta neste trabalho aplica-se a casos com estas características.

Outro aspecto que merece destaque é a contribuição desta pesquisa, que:

- Adapta uma modelagem de apoio à decisão para um problema de diagnóstico do grau de satisfação (são proble- máticas que apresentam fortes diferenças, que por serem sutis, nem sempre são percebidas);

- Incorpora, de forma inédita, um pré-processamento estatístico de dados ao método ELECTRE TRI. Isto possibilita:

- Um tratamento diferenciado para a estimativa dos parâmetros $p$ e $q$ (limites de preferência e de indiferença, respectivamente) - vale registrar que não se encontrou na extensa revisão bibliográfica efetuada qualquer tipo de justificativa matemática para a definição destes parâmetros que, sem exceção, são apresentados apenas como estimativas arbitrárias elaboradas pelos analistas;

Quadro 3: Classes de equivalência.

\begin{tabular}{|c|c|c|c|}
\hline CLASSES DE SATISFAÇÃO & DESCRIÇÃO DAS CLASSES & LIMITE SUPERIOR & LIMITE INFERIOR \\
\hline A & Alta & - & 4,5 \\
B & Muito Alta & 4,5 & 3,5 \\
C & Média & 3,5 & 2,5 \\
D & Baixa & 2,5 & 1,5 \\
E & Muito Baixa & 1,5 & - \\
\hline
\end{tabular}

Figura 3: Classes de referência e perfis adotados neste trabalho.

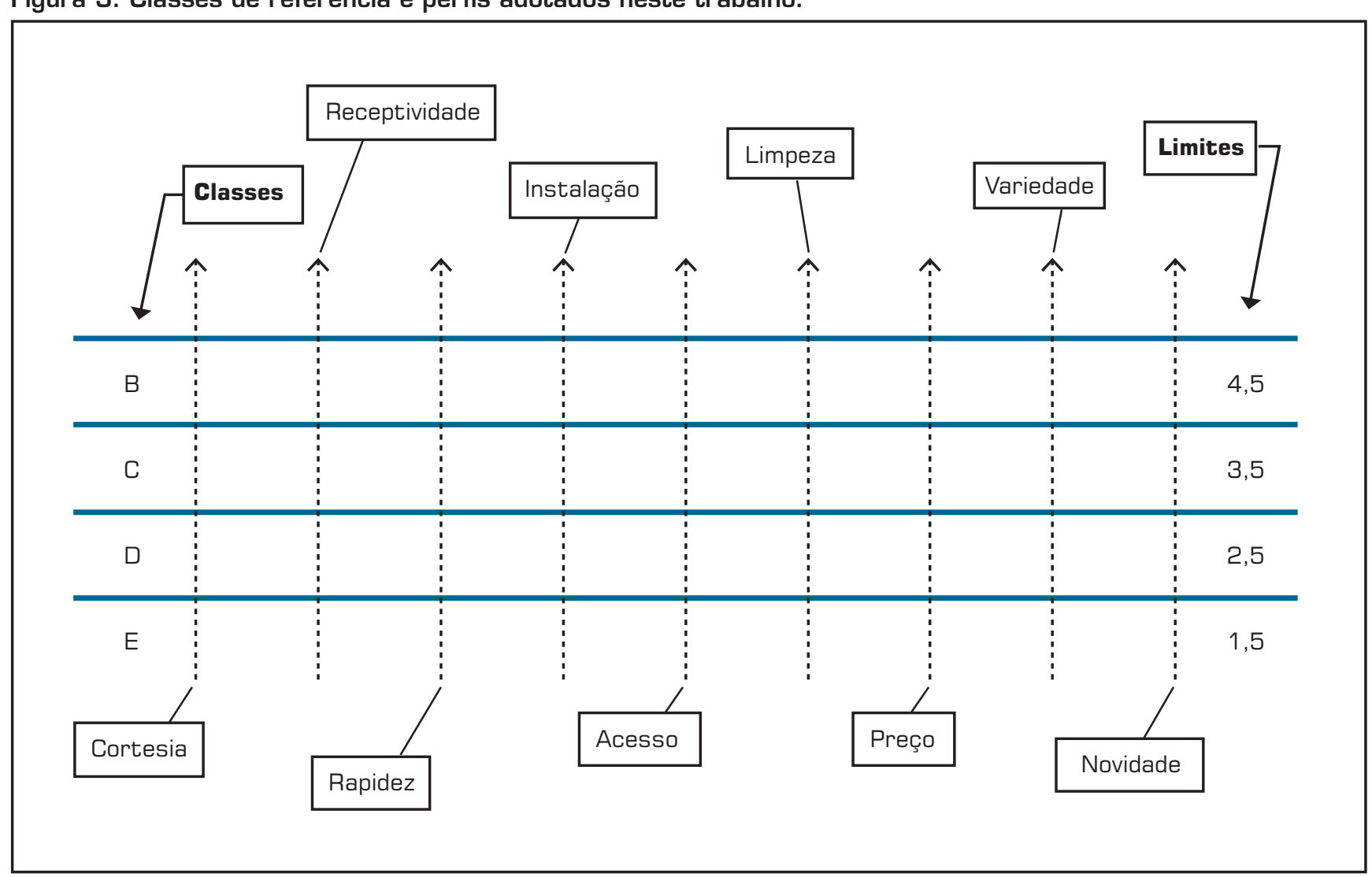


- Uma maior credibilidade no uso de um método de AMD monodecisor na resolução de um problema multidecisor;

- Trata, de forma inédita, a questão incomparabilidade, destacando que nem sempre a mesma é originadas por imperícias na modelagem ou nas avaliações de desempenho.

Uma importante diferença em relação aos métodos tradicionais de avaliação de desempenho é que a classificação obtida pelo serviço independe do valor alcançado pelo mesmo na média ponderada. Isto elimina efeitos compensatórios que podem mascarar a classificação e torna o resultado independente do valor numérico associado à escala de julgamento do desempenho. No caso da média ponderada, o "fundo de escala" funciona como "peso" para o critério.

Apesar da aplicação da abordagem proposta depender do uso de um método não muito difundido no meio organizacional, o Método ELECTRE TRI, este não foi um fator prejudicial a sua aplicabilidade na modelagem desenvolvida - empresa de pequeno porte, atuante no setor de varejo (loja) em um shopping.

O modelo apresentado tem o objetivo central de ilustrar a aplicação da abordagem proposta, não devendo ser tomado como referência de modelo geral para avaliação do grau de satisfação dos clientes. Por exemplo: com o apoio de uma revisão bibliográfica, o "dono do negócio" definiu o conjunto de critérios que ele considerava crítico e para o qual ele gostaria de ter a percepção do consumidor sobre o seu negócio. Ou seja: é um modelo particular, adequado a uma determinada situação. Este fato não invalida a proposta deste trabalho, nem tampouco implica em perda de generalidade, pois a proposta central deste trabalho não é o modelo, mas sim a modelagem.

Seguindo esta observação, experimentos e estudos de aplicação devem ser realizados abordando diferentes tipos de serviços e verificando o comportamento dos resultados e da abordagem aqui proposta em diferentes situações. Neste sentido vale observar que o número de referências na literatura sobre "aplicações abordagens multicritério" é ainda muito inferior ao de aplicações de outros instrumentos de gestão.

ANEXO I: Resultados obtidos.

\begin{tabular}{|l|l|l|l|}
\hline CONSUMIDOR & $\begin{array}{c}\text { GLASSIFICACGÄO PESSIMISTA } \\
\text { (MAIS EXIGENTE) }\end{array}$ & $\begin{array}{c}\text { GLASSIFICACGÄ0 OTIMISTA } \\
\text { (MENOS EXIGENTE) }\end{array}$ & INGOMPARABILIDADE \\
\hline C1 & A & A & N \\
\hline C2 & B & A & S \\
\hline C3 & B & A & S \\
\hline C4 & A & A & N \\
\hline C5 & A & A & N \\
\hline C6 & A & A & N \\
\hline C7 & A & A & N \\
\hline C8 & A & A & N \\
\hline C9 & A & A & N \\
\hline C10 & A & A & N \\
\hline C11 & A & A & N \\
\hline C12 & A & A & N \\
\hline C13 & B & A & S \\
\hline C14 & A & A & N \\
\hline C15 & A & A & N \\
\hline C16 & A & A & N \\
\hline C17 & A & A & N \\
\hline C18 & A & A & N \\
\hline C19 & A & A & N \\
\hline C20 & A & A & N \\
\hline & & & \\
\hline
\end{tabular}




\begin{tabular}{|c|c|c|c|}
\hline C21 & A & A & $\mathrm{N}$ \\
\hline C22 & A & A & $N$ \\
\hline C23 & A & A & $\mathrm{N}$ \\
\hline C24 & A & A & $\mathrm{N}$ \\
\hline C25 & A & A & $N$ \\
\hline C26 & A & A & $\mathrm{N}$ \\
\hline $\mathrm{C} 27$ & $A$ & A & $\mathrm{N}$ \\
\hline C28 & $A$ & A & $\mathrm{N}$ \\
\hline C29 & A & A & $\mathrm{N}$ \\
\hline СЗО & $A$ & A & $\mathrm{N}$ \\
\hline С31 & $A$ & A & $\mathrm{N}$ \\
\hline сз2 & $A$ & A & $\mathrm{N}$ \\
\hline С33 & $A$ & A & $\mathrm{N}$ \\
\hline C34 & $A$ & A & $\mathrm{N}$ \\
\hline C35 & $A$ & A & $\mathrm{N}$ \\
\hline С36 & $A$ & A & $N$ \\
\hline C37 & $A$ & A & $\mathrm{N}$ \\
\hline С38 & $A$ & $A$ & $\mathrm{~N}$ \\
\hline С39 & A & A & $\mathrm{N}$ \\
\hline C40 & $A$ & $A$ & $\mathrm{~N}$ \\
\hline C41 & $A$ & A & $\mathrm{N}$ \\
\hline C42 & B & A & $\mathbf{s}$ \\
\hline C43 & B & A & 5 \\
\hline C44 & B & A & $\mathbf{s}$ \\
\hline $\mathrm{C45}$ & A & A & $\mathrm{N}$ \\
\hline C46 & B & A & $\mathbf{s}$ \\
\hline C47 & B & A & $\mathbf{s}$ \\
\hline C48 & C & A & $\mathbf{s}$ \\
\hline C49 & B & A & $\mathbf{s}$ \\
\hline C50 & B & A & $\mathbf{s}$ \\
\hline C51 & B & A & $\mathbf{s}$ \\
\hline C52 & B & A & $\mathbf{s}$ \\
\hline C53 & A & $A$ & $\mathrm{~N}$ \\
\hline C54 & $B$ & $B$ & $\mathrm{~N}$ \\
\hline C55 & B & A & $\mathbf{s}$ \\
\hline C56 & A & $A$ & $\mathrm{~N}$ \\
\hline C57 & $A$ & $A$ & $\mathrm{~N}$ \\
\hline C58 & A & A & $\mathrm{N}$ \\
\hline C59 & $A$ & $A$ & $\mathrm{~N}$ \\
\hline C60 & B & A & $\mathbf{N}$ \\
\hline
\end{tabular}




\begin{tabular}{|l|l|l|l|}
\hline C61 & B & A & A \\
\hline C62 & A & A & N \\
\hline C63 & A & A & N \\
\hline C64 & B & A & S \\
\hline C65 & B & A & S \\
\hline C66 & A & A & N \\
\hline C67 & B & A & S \\
\hline C68 & B & A & S \\
\hline C69 & B & A & S \\
\hline C70 & B & A & S \\
\hline C71 & B & A & S \\
\hline C72 & B & A & S \\
\hline DELICIAS & A & A & A \\
\hline
\end{tabular}

Nota: DELÍCIAS representa um avaliador cujos julgamentos seriam a média dos julgamentos dos 72 avaliadores consultados.

\section{Artigo recebido em 04/02/2005 Aprovado para publicação em 18/04/2007}

\section{- Referências}

ANTUNES, H. A.; ALVES, M. J.; SILVA, A. L.; CLÍMACO, J. N. Algumas reflexões sobre uma base de métodos fde programação linear multicritério. Investigação Operacional, v. 9, n. 2, p. 19-35. 1989.

ARROW, K. J. Social Choice and Individual Values. London: JohnWiley and Sons, 1963.

AZEVEDO, M. C. Avaliação estratégica da competitividade: abordagem multicritério através do ELECTRE TRI. Dissertação (Mestrado em Engenharia de Produção), Universidade Estadual do Norte Fluminense Darcy Ribeiro (UENF), Campos, 2001.

BANA; COSTA, C. Introdução geral às abordagens multicritério de apoio à tomada de decisão. Investigação operacional, v. 5, n. 1, p. 117-139, jun. 1988 .

MEMSOR - Método Multicritério para Segmentação Ordenada. Investigação operacional, v. 10, n. 1, p. 19-28, jun. 1990.

BARROSO, M. F. C. M. Análise multicritério e avaliação de desempenho docente sob a ótica do corpo discente em instituições de ensino superior. Dissertação (Mestrado em Engenharia de Produção), Universidade Estadual do Norte Fluminense Darcy Ribeiro (UENF), Campos dos Goytacazes, 2002.
BATOCCHIO, A.; BIAGIO, L. A. A importância da avaliação do capital intelectual na administração estratégica. XIX ENEGEP (Encontro Nacional de Engenharia de Produção). Universidade Federal do Rio de Janeiro (UFRJ), Rio de Janeiro, Brasil: Associação Brasileira de Engehria de Produção (ABEPRO), 1999. 17 p.

BOLJWIN, P. T.; KUMPE, T. Manufacturing in the 1990's - Productivity, Flexibility and Innovation. Long Range Planning, v. 23, n. 4 , p. $44-57,1990$.

CHANKONG, Y.; HAIMES, Y. Multiobjective Decision Making. Amsterdam: Ed. North Holland, 1983. p.

CORRÊA, P. S. Proposta de Aplicação do Método de Análise Hierárquica (AHP) em Pesquisa de Avaliação Pós-Ocupação (APO). Dissertação (Mestrado Profissional em Sistemas de Gestão), Universidade Estadual do Norte Fluminense Darcy Ribeiro (UENF), Campos dos Goytacazes, 2004. $159 \mathrm{p}$.

COSTA, H. G. An multicriteria approach to evaluate consumer satisfaction: a contribution to marketing. VIII International Conference on Decision Support Systems (ISDSS'05). Porto Alegre, RS, Brasil: International Society of Decision Suppotrt Systems, 2005. 10 p.
COSTA, H. G.; FREITAS, A. L. P. Metodologia multicritério para a medição e classificação do grau de satisfação de clientes. METROLOGIA 2000 (International Conference on Advanced Metrology). São Paulo, SP: Brazilian Society of Metrology, 2000. 8 p.

DALLY, J. W.; RILEY, W. F. Experimental Stress Analysis. New York, USA: McGrawHill, 1998.

DENTON, K. Qualidade em serviços: o atendimento ao cliente como fator de vantagem competitiva. São Paulo, Brasil: Ed. Makron Books, 1991

FISHBURN, P. C. Utility theory for decision making. New York: Wiley, 1970.

FREITAS, A. L. P. Avaliação e Classificação da Qualidade em Serviços: uma abordagem multicritério. Dissertação (Mestrado Profissional em Sistemas de Gestão), Universidade Estadual do Norte Fluminense Darcy Ribeiro (UENF), 1997.

FREITAS, A. L. P.; COSTA, H. G. Uma abordagem multicritério para avaliação e classificação de serviços. Gestão e Produção, v. 5, n. 3, p. $272-283,1998$.

GRIGOROUDIS, P.; SISKOS, Y. Preference disaggregation for measuring and analyzing customer satisfaction: The MUSA method. European Journal of Operational Research, v. 143, n. 100, p. 148-170, 2001.
GRÖNROSS, C. Service manegement and Marketing. Toronto, Canadá: Ed. Lexington Books, 1990

HERRERA, W. D. M.; COSTA, H. G. Contribuições da análise multicritério à determinação do grau de proximidade em arranjos físicos. Produto \& Produção, 2001

HWANG, C.; YOON, K. Multiple attribute decision making: Methods and application. New York: Springer, 1981.

KEENEY, R. L.; RAIFFA, H. Decisions with Multiple Objectives: preferences and value tradeoffs New York: John Willey \& Sons, 1976, 569 p.

MANSUR, A. F. U. Aplicação da metodologia ELEQUAL para a avaliação da qualidade de serviços: um estudo de caso. Dissertação (Mestrado em Engenharia de Produção), Universidade Estadual do Norte Fluminense Darcy Ribeiro, Campos dos Goytacazes, Brasil, 2000.

MANSUR, A. F.; COSTA, H. G. Aplicação da metodologia ELEQUAL para avaliação da qualidade em serviços: um estudo de caso XX Encontro Nacional de Engenharia de Produção. Salvador, Brasil: Associação Brasileira de Engenharia de Produção (ABEPRO), 2001. 


\section{- Referências}

MIHELIS, G.; et al. Customer satisfaction measurement in the private bank sector European Journal of Operational Research, v. 130 , p. 347360,1998

MONTANA, P. J.; CHARNOV, B. H. Administração. São Paulo, Brasil: Ed. Saraiva, 1998.

MOUSSEAU, V.; SLOWINSKI, R. ZIELNIEWICZ. ELECTRE TRI 2.0a. methodological guide and user's manual. Document du LAMSADE. Université de Paris-Dauphine. Paris: Fev. 1999.

POLITIS, Y.; SISKOS, Y. Multicriteria methodology for the evaluation of a Greek engineering department. European Journal of Operational Research, v. 156, n. 1, p. 223-240, 2004

RIBEIRO, T. A. C. Avaliação institucional de IES: um estudo de caso sob a ótica do corpo discente. Dissertação (Mestrado em Engenharia de Produção), Universidade Estadual do Norte Fluminense "Darcy Ribeiro" (UENF), Campos dos Goytacazes, 2003. $125 \mathrm{p}$
RIBEIRO, T. A. C.; COSTA, H. G. Aplicação do método ELECTRE TRI à classificação da percepção do desempenho de IES por parte do corpo discente. ENCONTRO NACIONAL DE ENGENHARIA DE PRODUÇÃO (ENEGEP 2005). Porto Alegre, RS: Associação Brasileira de Engenharia de Produção (ABEPRO), 2005. 8 p.

ROY, B. Classement et choix en présence de points de vue multiples (la méthode ELECTRE). Lausanne Presses Polytechniques et Universitaires Romandes, 1968.

. ELECTRE III: Un alghoritme de methode de classements fonde sur une representation floue des préférences en presence de critères multiples. Cahieres de CERO, v. 20, n. 1, p. 3-24, 1978.

ROY, B.; BERTIER, P. M. La méthode ELECTRE II: Une méthode de classement en présence de critéres multiples. Paris: SEMA (Metra International) p. 45, 1971
ROY, B.; BOYSSOU, D. Méthodologie Multicritère d'Aide à la Décision. Paris: Ed. Economica, 1985.

ROY, B. M.; SKALKA, J. ELECTRE IS: Aspécts methodologiques et guide d'utilization. Cahier du LAMSADE. Université de Paris-Dauphine. Paris: Fev. 1985.

ROY, B.; HUGONNARD, J. C. Classement des prolongements de lignes de stations en banlieu parisienne. Cahiers u LAMSADE. Université Dauphine et RATP. Paris, 1981

SAATY, T. L. The Analytic Hierarquic Process. Pittsburg: RWS Publications, 1980.

SILVA, R. B. Medição da satisfação do cliente quanto a serviços prestados levando em consideração a expectativa por ele trazida. XVIII ENEGEP (Encontro Nacional de Engenharia de Produção). Universidade Federal Fluminense (UFF), Niterói, Brasil Associação Brasileira de Engenharia de Produção (ABEPRO), 1998. 8 p.
SISKOS, Y. et al. Measuring custome satisfaction using a collective preference disaggregation model. Journal of Global Optimization, v. 12, p. 175-195. 1998.

TSAUR, S.H.; CHANG, T.Y. e YEN, C.H. The evaluation of airline service quality by fuzzy MCDM. Tourism Management, v. 23, p. 107-115. 2002.

VINCKE, Ph. Multicriteria decision Aid. New York: John Wiley, 1992.

WEST, T. M.; HUANG, K. J. A spreadsheet-based methodology for quality evaluations. Computers and Industrial Enginnering, v. 29, n. 1, p. 49-53. 1995.

YU, W. ELECTRE TRI - Aspects Methodologiques et Guide d'Utilisation. Document du LAMSADE. Université de Paris-Dauphine. Paris, 1992

ZELENY, M. Multiple Criteria Decision Making. New York: McGraw-Hill, 1982.

\section{- Agradecimentos}

Este trabalho foi viabilizado pelo apoio institucional da Universidade Federal Fluminense, além do apoio financeiro do Conselho Nacional de Desenvolvimento Científico e Tecnológico (CNPq).

\section{Sobre os autores}

Helder Gomes Costa, D. Sc.

Universidade Federal Fluminense (UFF)

End.: Rua Passo da Pátria, 156, sala 309 - São Domingos - Niterói - RJ - 24210-240

Tel.: (21) 2629-5478 / 5433 / 2629-5432 Fax: (21) 2629-5435

E-mail: hgc@vm.uff.br

\section{André Fernando Uébe Mansur, M.Sc.}

Centro Federal de Educação Tecnológica (CEFET-Campos)

End.: Rua Dr. Siqueira, 273 - Parque Dom Bosco - Campos dos Goytacazes - RJ - 28030-130

Tel.: (22 ) 2726-2800

E-mail: uebe@hotmail.com

\section{André Luís Policani Freitas, D.Sc.}

Universidade Estadual do Norte Fluminense (UENF)

End.: Av. Alberto Lamego, 2000, Bloco-CCT - Horto - Campos dos Goytacazes - RJ - 28013-600

Tel.: (21) 2726-1632

E-mail: policani@uenf.br

\section{Rogério Atem de Carvalho, D.Sc.}

Centro Federal de Educação Tecnológica (CEFET-Campos)

End.: Rua Dr. Siqueira, 273 - Parque Dom Bosco - Campos dos Goytacazes - RJ - CEP 28030-130

Tel.: (22 ) 2726-2800

E-mail: ratem@cefetcampos.br 